\title{
Thrombosis recanalization by paeoniflorin through the upregulation of urokinase-type plasminogen activator via the MAPK signaling pathway
}

\author{
SONGSHAN YE, BINGYU MAO, LEI YANG, WEIYUN FU and JUNRAN HOU \\ Department of Medical Experimental Center, Zhang Zhongjing Traditional Chinese Medicine College, \\ Nanyang Institute of Technology, Nanyang, Henan 473004, P.R. China
}

Received May 22, 2015; Accepted April 6, 2016

DOI: $10.3892 / \mathrm{mmr} .2016 .5146$

\begin{abstract}
Paeoniflorin, the major component of Paeonia lactiflora pall, has previously been reported to prevent thrombosis. Plasminogen activator urokinase (uPA) is a serine protease that markedly facilitates normal thrombosis resolution. Paeoniflorin and uPA have been linked to the mitogen-activated protein kinase (MAPK) signaling pathway. In the current study, the influence of paeoniflorin on the expression of uPA was investigated and the underlying regulatory mechanism was preliminarily determined. The prothrombotic state of the model animals treated with paeoniflorin were assessed by enzyme-linked immunosorbent assay (ELISA). Additionally, the cytotoxicity of paeoniflorin on human umbilical vein endothelial cell (HUVEC) cultures was estimated using a methyl thiazolyl tetrazolium assay and the possible pathways involved in the interaction between paeoniflorin and uPA were evaluated using western blot analysis. The ELISA results demonstrated that the levels of 6-keto prostaglandin $\mathrm{F}_{1 \mathrm{a}}$, fibronectin and uPA were significantly upregulated by treatment with paeoniflorin compared with control $(\mathrm{P}<0.05)$. By contrast, the expression of fibrinogen, D-dimer and thromboxane $\mathrm{B}_{2}$ were inhibited. With an increase in the concentration of paeoniflorin the cell viability of HUVECs decreased gradually. The results of western blot analysis demonstrated that paeoniflorin increased the phosphorylation of MAPK 14 (p38) and MAPK 8 (JNK). The present study demonstrated that paeoniflorin has the potential to improve the prethrombotic state and recanalize thrombosis by increasing the expression of UPA, which may be mediated via regulation of the $\mathrm{p} 38$ and JNK MAPK signaling pathways.
\end{abstract}

Correspondence to: Professor Bingyu Mao, Department of Medical Experimental Center, Zhang Zhongjing Traditional Chinese Medicine College, Nanyang Institute of Technology, 80 Changjiang Road, Nanyang, Henan 473004, P.R. China

E-mail: bingyumao2005@sina.com

Key words: prothrombotic states, paeoniflorin, plasminogen activator urokinase, mitogen-acitvated protein kinase
However, this treatment effect was dependent on the concentration of paeoniflorin used, an unsuitable concentration of the agent would result in a negative effect on the anti-thrombosis pathways.

\section{Introduction}

Platelets are anucleated blood cells released from bone marrow megakaryocytes through a complex process that is not fully understood $(1,2)$. At sites of vascular injury, platelets come into contact with exposed subendothelial components and form a plug to prevent excessive blood loss. However, if this process is not controlled, it may result in thrombotic events causing severe, life-threatening diseases, including myocardial infarction or ischemic stroke $(3,4)$. Based on the above evidence, thrombosis is almost exclusively treated by anti-platelet and anti-coagulant compounds, which reduce thrombosis propagation. However, current anti-platelet and anticoagulant drugs always have poor efficacy and associated side effects, these limitations render it imperative to develop more effective drugs with reduced side effects (5-8).

A previous study by Northeast et al (9) using a rat model of venous thrombosis demonstrated that during natural resolution of venous thrombi, the activity of plasminogen activator urokinase (uPA) is upregulated (9). Subsequent gene level studies demonstrated that deletion of the gene encoding uPA markedly inhibited normal thrombosis resolution (10), while the adenoviral expression of uPA enhanced venous thrombosis resolution (11). These noteworthy results suggest there may be clinical potential in upregulating the expression of uPA in thrombosis resolution. UPA is a serine protease that is important for cell migration and angiogenesis $(12,13)$. Several previous studies demonstrated that the secretion of uPA was increased by the overexpression of mitogen-activated protein kinase (MAPK) 14 (p38) and MAPK8 (JNK) (14-16), which are important components of the MAPK signaling pathway. Activation of the pathway is associated with the activation of various transcription factors, resulting in the increased expression of numerous genes involved in tumor cell proliferation, apoptosis and angiogenesis (15). Thus, it is possible that the p38 and JNK MAPK pathways participate in the process of uPA-regulated thrombosis recanalization, 
and that agents with an effect on this pathway may improve thrombosis treatment.

The herb Paeonia lactiflora pall, which is known as 'Shao Yao' in Chinese, has been used in traditional Chinese medicine for over 1,000 years to treat cramp, pain, giddiness and congestion (17). Paeoniflorin, the major component of Paeonia lactiflora pall, has previously been reported to exhibit various pharmacological effects, including anti-inflammation, anti-allergy, anti-hyperglycemia, enhanced cognition and thrombosis prevention (18). Furthermore, paeoniflorin was also reported to have an effect on MAPK pathways (18). These previous studies suggest that paeoniflorin may regulate uPA to promote recanalization following thrombosis.

To verify the hypothesis, the current study measured the concentration of fibronectin (FN), fibrinogen (FIB), D-dimer (D-D), 6-ketoprostaglandin $\mathrm{F}_{1 \mathrm{a}}\left(6-\mathrm{Keto}_{\mathrm{PGF}} \mathrm{PG}_{1 \mathrm{a}}\right.$ ), thromboxane $\mathrm{B}_{2}$ $\left(\mathrm{TXB}_{2}\right)$ and $\mathrm{uPA}$ in the serum of spontaneously hypertensive rats (SHRs) using a sandwich enzyme-linked immunosorbent assay (ELISA) to confirm the effect of paeoniflorin on thrombosis recanalization. The cytotoxicity of paeoniflorin on human umbilical vein endothelial cells (HUVECs) was estimated by 3-(4,5-dimethylthiazol-2-yl)-2,5-diphenyltetrazolium bromide (MTT) assay and the potential link between paeoniflorin and uPA was evaluated using western blot analysis. The present study aimed to determine the mechanism by which the effects of paeoniflorin on thrombosis are mediated and to improve the practical use of this Chinese medicine compound in the clinic. The results of the present study demonstrated the preliminary mechanism of paeoniflorin to enhance the expression of uPA to recanalize thrombosis, which may facilitate uPA as a potential therapeutic strategy against thromobosis in the clinic.

\section{Materials and methods}

Materials. Paeoniflorin (purity, >99\%; Fig. 1) was obtained from Sigma-Aldrich (St. Louis, MO, USA). Antibodies obtained for use in the present study are as follows: Rabbit monoclonal anti-JNK (cat. no. ab110724), rabbit polyclonal anti-phosphorylated (p)-JNK (cat. no. ab47337), rabbit monoclonal anti-p38 (cat. no. ab170099), rabbit polyclonal anti-p-p38 (cat. no. ab47363), rabbit monoclonal anti-uPA (cat. no. ab133563) and rabbit polyclonal anti-glyceraldehyde 3-phosphate dehydrogenase (GAPDH; cat. no. ab9485) were purchased from Abcam (Cambridge, MA, USA). JNK inhibitor (SP600125) and p38 inhibitor (SB203580) were obtained from EMD Millipore (Billerica, MA, USA). The HUVEC line was purchased from the American Type Culture Collection (Manassas, VA, USA). The cells were cultured in medium 199 (Gibco; Thermo Fisher Scientific, Inc., Waltham, MA, USA) supplemented with $20 \%$ fetal bovine serum (Gibco; Thermo Fisher Scientific, Inc.), 2 mM L-glutamine (Sigma-Aldrich), $5 \mathrm{U} / \mathrm{ml}$ heparin (Sigma-Aldrich), $100 \mathrm{IU} / \mathrm{ml}$ penicillin (Sigma-Aldrich), $10 \mu \mathrm{g} / \mathrm{ml}$ streptomycin (Sigma-Aldrich) and $50 \mu \mathrm{g} / \mathrm{ml}$ endothelial cell growth supplement (American Type Culture Collection). Newly purchased cells were cultured in a humidified incubator at $5 \% \mathrm{CO}_{2}$ and $37^{\circ} \mathrm{C}$. Cells between passage 3 and 6 were used for further experiments.

Grouping of model animals and gavage experiment. Male SHRs ( $n=30$; age 18 weeks; weight, 350-400 g) were provided

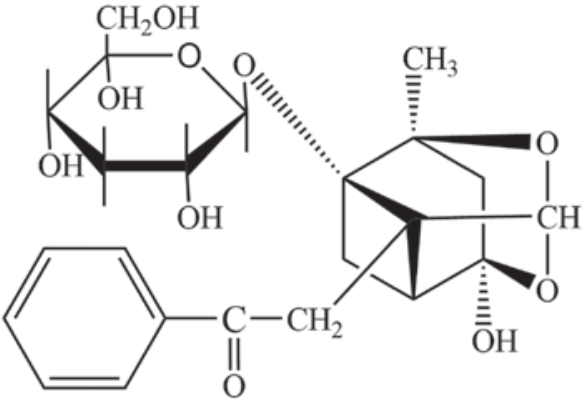

Figure 1. Chemical structure of paeoniflorin.

by the Laboratory Animal Center of the First Affiliated Hospital of Sun Yat-Sen University (Guangzhou, China) for the thrombosis model and were housed in cages at room temperature $\left(20-25^{\circ} \mathrm{C}\right)$ and $80 \%$ humidity, with a 12 -h light/dark cycle. Food and water were available ad libitum. The animals were randomly divided into control, paeoniflorin and aspirin groups ( $n=10$ per group). The control, paeoniflorin and aspirin group animals received $2 \mathrm{ml} / \mathrm{kg}$ normal saline, $5 \mathrm{mg} / \mathrm{kg}$ paeoniflorin and $50 \mathrm{mg} / \mathrm{kg}$ aspirin (Sigma-Aldrich), respectively, every 2 days for 2 weeks by gavage method. All the animal experiments were conducted in accordance with the Guide for the Care and Use of Laboratory Animals published by the National Institutes of Health (19), and the present study was approved by the ethics committee of Nanyang Institute of Technology (Nanyang, Henan).

Collection of serum samples and ELISA. Each rat was anesthetized with $10 \%$ chloral hydrate $(0.3 \mathrm{ml} / 100 \mathrm{~g}$; Sigma-Aldrich) at the end of the treatment by intraperitoneal injection, the rats were sacrificed and blood samples were collected from the abdominal aorta by opening the abdominal cavity. Serum was separated by centrifugation at 2,200 $\mathrm{xg}$ at $4^{\circ} \mathrm{C}$ for $20 \mathrm{~min}$ and preserved at $-80^{\circ} \mathrm{C}$. For each serum sample, the concentration of FN, FIB, D-D, 6-Keto-PGF ${ }_{1 \mathrm{a}}, \mathrm{TXB}_{2}$ and $\mathrm{uPA}$, which were all closely associated with the prothrombotic state (PTS), were measured using ELISA kits according to the manufacturer's instructions (Nanjing Jiancheng Bioengineering Institute, Nanjing, China). Briefly, plates were coated and incubated overnight with $3.4 \mathrm{mg} / \mathrm{ml}$ non-biotinylated 3D5 (100 $\mu \mathrm{l} /$ well; Nanjing Jiancheng Bioengineering Institute) in $200 \mathrm{mM}$ $\mathrm{NaHCO}_{3}$ at $\mathrm{pH} 9.6$ at $4^{\circ} \mathrm{C}$, and then washed 4 times with phosphate-buffered saline (PBS) containing $0.05 \%$ Tween 20 (PBST). Following incubation with blocking buffer ( $150 \mu \mathrm{l} /$ well PBST containing $2.5 \%$ gelatin) for $2 \mathrm{~h}$ at $37^{\circ} \mathrm{C}$, the plates were again washed 4 times with PBST, and a $100 \mu$ l serum sample (diluted 1:1 with PBS) was added to each well. The plates were then incubated at $37^{\circ} \mathrm{C}$ for $2 \mathrm{~h}$. After washing 4 times with PBST, $100 \mu \mathrm{l}$ of $1 \mu \mathrm{g} / \mathrm{ml}$ biotinylated 3D5 in blocking buffer was added to each well and incubated at $37^{\circ} \mathrm{C}$ for another $2 \mathrm{~h}$. The wells were then washed 4 times with PBST and incubated with $100 \mu \mathrm{l} /$ well ExtrAvidin-Alkaline phosphatase (Sigma-Aldrich) in blocking buffer $(1: 5,000)$ and incubated for $1 \mathrm{~h}$ at $37^{\circ} \mathrm{C}$. Following another 4 washes with PBST, the enzyme substrate Yellow 'pNPP' (100 $\mu \mathrm{l} /$ well; Sigma-Aldrich) was added to each well and incubated for $30 \mathrm{~min}$ at $37^{\circ} \mathrm{C}$ for 

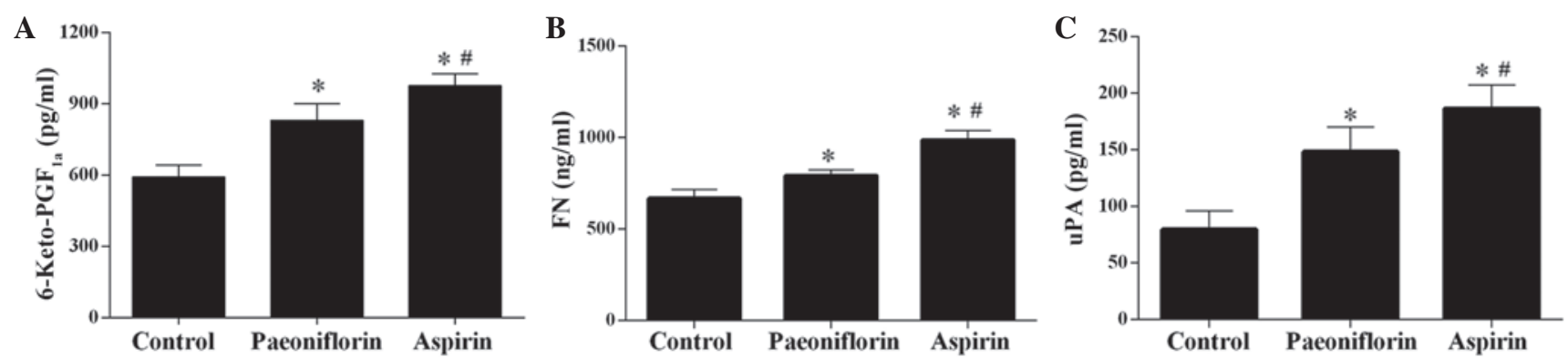

Figure 2. Effect of paeoniflorin treatment on the levels of 6-Keto-PGF ${ }_{1 a}$, FN and uPA. The levels of (A) 6-Keto-PGF upregulated in the serum of model rats. Values are expressed as the mean \pm standard deviation. ${ }^{*} \mathrm{P}<0.05$ vs. the control group, ${ }^{\#} \mathrm{P}<0.05$ vs. the paeoniflorin group. 6-Keto-PGF $1 \mathrm{a}$, 6-keto prostaglandin $\mathrm{F}_{\text {1a }}$; FN, fibronectin; uPA, plasminogen activator urokinase.

color development. Optical density (OD) values of different targeted molecules were recorded with a microplate reader at a wavelength of $450 \mathrm{~nm}$ (Model 550; Bio-Rad Laboratories, Inc., Hercules, CA, USA). The concentration of each protein was estimated from the standard curve determined by serial dilution.

Cytotoxicity of paeoniflorin and cell proliferation assay. To determine the cytotoxic effect of paeoniflorin on HUVECs, a 3-(4,5dimethylthiazol-2-yl)-2,5-diphenyltetrazolium bromide (MTT) assay was performed according to the standard protocol. Briefly, $50 \mu 1$ exponentially growing HUVECs $\left(2 \times 10^{5}\right.$ cells $\left./ \mathrm{ml}\right)$ were seeded into a 96 -well plate in triplicate. The cells were treated with 0 (control), 0.01, 0.05, 0.1, 0.5, 1, 5, 10, 20 and $50 \mu \mathrm{mol} / 1$ paeoniflorin for $24 \mathrm{~h}$. Following incubation with paeoniflorin, $5 \mathrm{mg} / \mathrm{ml}$ MTT (Sigma-Aldrich) was added to each well and incubated for another $4 \mathrm{~h}$ at $37^{\circ} \mathrm{C}$. The OD values in different wells were recorded using a microplate reader at $450 \mathrm{~nm}$. The survival rates (\%) of cells with the different treatments were calculated as: (OD value in treatment group - OD value in blank control group)/(OD value in negative control group - OD value in blank control group) x 100 .

The highest concentration with the majority cells surviving was $0.5 \mu \mathrm{mol} / 1$, thus, the cell proliferation assay was conducted using this concentration. HUVECs were treated with $5 \mu \mathrm{mol} / 1$ paeoniflorin for $0,24,48,72$ and $96 \mathrm{~h}$. Normal HUVECs were used as a control to determine the effect of paeoniflorin on the viability of HUVECs over the time course. The detection of cell proliferation was conducted as described above. The OD values in different wells were recorded using the microplate reader at $450 \mathrm{~nm}$.

Western blot analysis. HUVECs were divided into 4 treatment groups: Untreated; incubated with $0.5 \mu \mathrm{mol} / 1$ paeoniflorin for $24 \mathrm{~h}$; pretreated with $50 \mu \mathrm{mol} / 1 \mathrm{SB} 203580$ for $1 \mathrm{~h}$ and incubated with $0.5 \mu \mathrm{mol} / 1$ paeoniflorin for $24 \mathrm{~h}$; and pretreated with $50 \mu \mathrm{mol} / 1 \mathrm{SP} 600125$ for $1 \mathrm{~h}$ and incubated with $0.5 \mu \mathrm{mol} / 1$ paeoniflorin for $24 \mathrm{~h}$. The protein samples were extracted from the cells after the 24-h incubation using SDS Lysis Buffer on ice for $30 \mathrm{~min}$. The protein concentration was determined using the Pierce BCA Protein assay kit (Thermo Fisher Scientific, Inc.). All the extracts were boiled with loading buffer for $5 \mathrm{~min}$ prior to sodium dodecyl sulfate polyacrylamide gel electrophoresis (SDS-PAGE) on 10\% gels (EMD Millipore). Proteins then were transferred onto polyvinylidene difluoride membranes (EMD Millipore). The membranes were washed with Tris-buffered saline-0.05\% Tween 20 (Sigma-Aldrich) three times for $20 \mathrm{~min}$ each time. The membranes were blocked with $5 \%$ non-fat milk prior to incubation with specific antibodies [JNK (1:1,000), p-JNK (1:800), p38 (1:800), p-p38 $(1: 1,000)$, uPA $(1: 1,000)$ and GAPDH $(1: 2,000)]$ overnight at room temperature. Following three additional washes, horseradish peroxidase-conjugated monoclonal goat anti-rabbit secondary antibodies (1:1,500; Beyotime Institute of Biotechnology; cat. no. A2080) were added and the membranes were incubated for another $4 \mathrm{~h}$. Following 3 final washes, the blots were developed using Beyo ECL Plus reagent (Beyotime Institute of Biotechnology) and the results were detected in the Chemi Doc ${ }^{\mathrm{TM}}$ MP system (Bio-Rad Laboratories, Inc.). GAPDH was used as a loading control for western blot analysis.

Statistical analysis. Data are expressed as the mean \pm standard deviation. Multiple comparisons were conducted using one-way analysis of variance with least significant difference test method. Statistical analysis was conducted using SPSS software version 19.0 (IBM SPSS, Armonk, NY, USA). P<0.05 was considered to indicate a statistically significant difference.

\section{Results}

Paeoniflorin improves the PTS and increases uPA expression in SHRs. The ELISA results demonstrated that treatment with paeoniflorin significantly increased the concentration of 6-Keto-PGF ${ }_{1 \mathrm{a}}, \mathrm{FN}$ and $\mathrm{uPA}$ in serum samples $(\mathrm{P}<0.05)$, however, the effect was weaker than that of aspirin, which served as a control as it is in anti-thrombotic drug (Fig. 2) (20). Furthermore, the levels of FIB, D-D and $\mathrm{TXB}_{2}$ were significantly downregulated by paeoniflorin, however, treatment with aspirin exerted a more potent effect (Fig. 3).

Effect of paeoniflorin on HUVEC viability and proliferation. The increasing paeoniflorin concentration had a negative effect on the cell viability of HUVECs (Fig. 4A). There was no significant difference in cell viability compared with control when the paeoniflorin concentration was $<0.5 \mu \mathrm{mol} / 1$, however, when the concentration was $>0.5 \mu \mathrm{mol} / 1$, the cell viability was significantly reduced. However, even at $50 \mu \mathrm{mol} / 1$ paeoniflorin, the cell viability remained within the safe range $(>70 \%)$.

Based on the results of the MTT assay, $0.5 \mu \mathrm{mol} / 1$ was determined as the suitable paeoniflorin concentration for use 

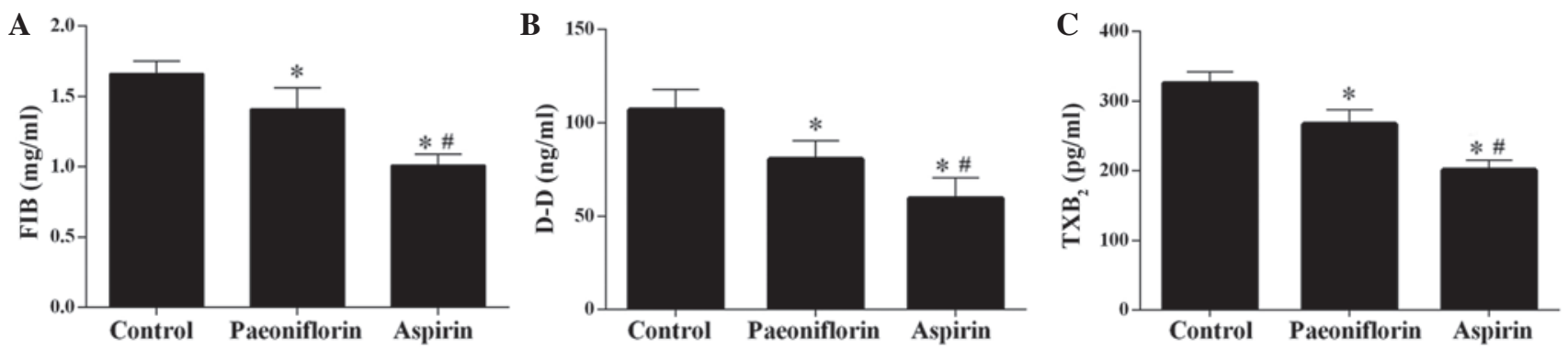

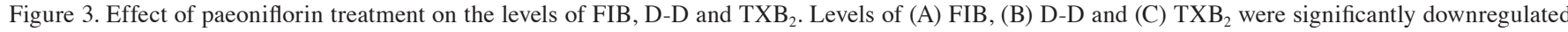
in the serum of model rats. Values are expressed as the mean \pm standard deviation. ${ }^{*} \mathrm{P}<0.05$ vs. the control group, ${ }^{*} \mathrm{P}<0.05$ vs. the paeoniflorin group. FIB, fibrinogen; D-D, D-dimer; $\mathrm{TXB}_{2}$, thromboxane $\mathrm{B}_{2}$.
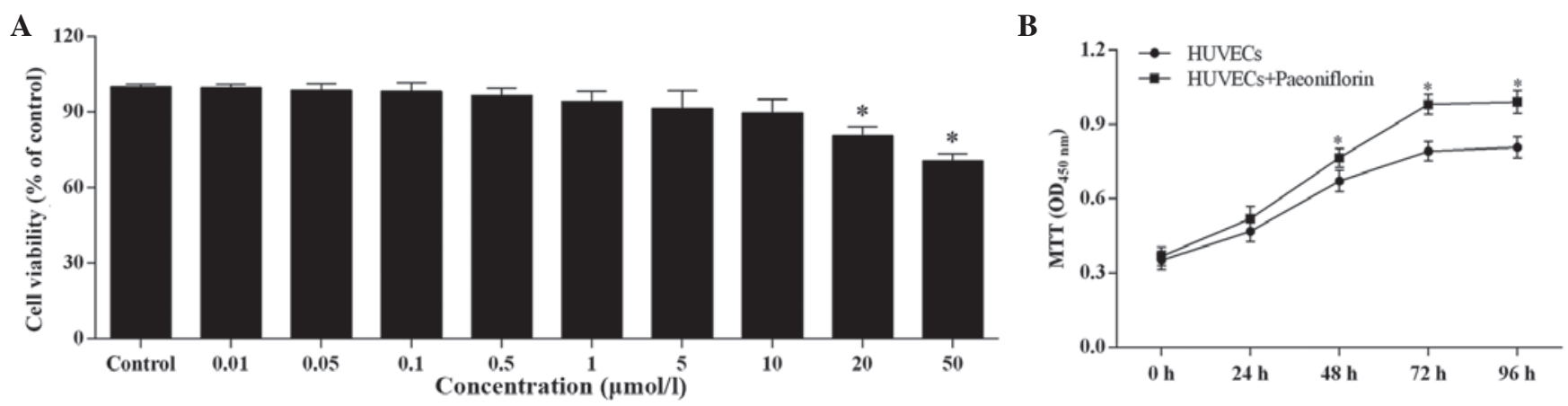

Figure 4. Effect of paeoniflorin on the proliferative ability of HUVECs. (A) The cells were incubated with the different concentrations of paeoniflorin for $24 \mathrm{~h}$. (B) HUVECs were incubated with $0.5 \mu \mathrm{mol} / 1$ paeoniflorin and the viability was measured at $0,24,48,72$ and $96 \mathrm{~h}$. The proliferative ability of HUVECs was improved compared with control HUVECs. Values are expressed as the mean \pm standard deviation. ${ }^{*} \mathrm{P}<0.05$ vs. (A) the control group and (B) the HUVECs. OD, optical density; HUVEC, human umbilical vein endothelial cell; MTT, 3-(4,5dimethylthiazol-2-yl)-2,5-diphenyltetrazolium bromide.
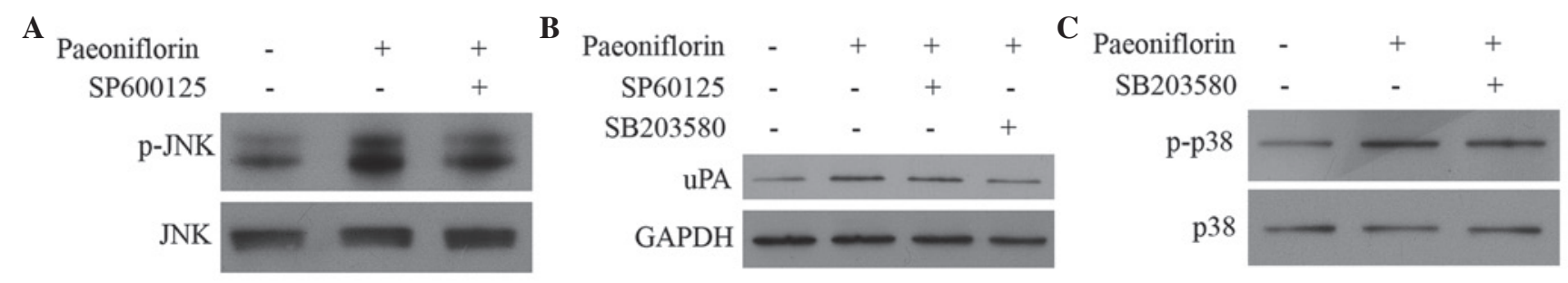

Figure 5. Effect of paeoniflorin on the anti-thrombus related pathway. Paeoniflorin increased the phosphorylation of (A) JNK, (B) the expression of uPA and (C) the phosphorylation of $\mathrm{p} 38$. JNK, mitogen-activated protien kinase 8; uPA, plasminogen activator urokinase; p-, phosphorylated; GAPDH, glyceraldehyde 3-phosphate dehydrogenase.

in further experiments. At this concentration, paeoniflorin significantly increased cell proliferation compared with untreated HUVECs after 48, 72 and 96-h treatment $(\mathrm{P}<0.05$; Fig. 4B).

Treatment of paeoniflorin activates the phosphorylation of $J N K$ and $p 38$, and upregulates the expression of $u P A$. To examine the effect of paeoniflorin on the MAPK pathway and the subsequent influence on the expression of uPA, the current study used western blot analysis to quantify the protein expression levels of JNK, p-JNK, p38, p-p38 and uPA. As demonstrated in Fig. 5, the expression levels of p-JNK, p-p38 and uPA in HUVECs were upregulated by paeoniflorin treatment compared with control. Treatment with SB203580 (p38 inhibitor) and SP600125 (JNK inhibitor) demonstrated direct effects on the MAPK pathway and synthesis of uPA.
Treatment with paeoniflorin activates the phosphorylation of JNK and p38 and upregulates the expression of uPA (Fig. 5). Treatment with SP600125, a JNK inhibitor, and SB203580, a p38 inhibitor, reduced the phosphorylation of JNK and p38, and inhibited the expression of uPA compared with paeoniflorin treatment (Fig. 5B and C).

\section{Discussion}

A previous study demonstrated that monocyte ingress in thrombosis resolution is dependent on the expression of uPA (21). It is hypothesized that uPA promotes thrombosis resolution by assisting monocyte recruitment to the thrombus; thus, facilitating revascularization and remodeling (22). However, application of uPA using a single adenoviral dose may not be an optimum treatment due to the larger volume 
of thrombi found in human veins, and the delivery of greater doses is currently expensive. In order to address this problem, a more effective application of uPA to recanalize thrombosis is required. Considering that the expression of $\mathrm{UPA}$ is regulated through MAPK pathways, the current study used agents that inhibit the pathway to investigate their effect on the associated processes.

The present study examined the effect of paeoniflorin, a compound used in Chinese medicine, on thrombosis and the expression of uPA. The results demonstrated that paeoniflorin improves the PTS in rat models of thrombosis and upregulates the expression of uPA via the MAPK signaling pathways. Ye et al (23) previously reported the antithrombotic effect of paeoniflorin, however, no further studies have been conducted to elucidate the underlying mechanism of this effect. In the current study, ELISA results demonstrated that the concentration of 6-Keto- $\mathrm{PGF}_{1 \mathrm{a}}$ and $\mathrm{FN}$ in serum samples was significantly increased, whereas the levels of FIB, $\mathrm{TXB}_{2}$, and DD were significantly reduced. 6-Keto-PGF Pa $_{1 \mathrm{a}}$ is the stable metabolite of prostaglandin $\mathrm{I}_{2}$. FN is important for stabilizing extracellular matrix material, and measuring the levels of FN may be used to assess endothelial damage $(24,25)$. FIB has previously been associated with an increased risk of venous thrombosis (26). $\mathrm{TXB}_{2}$ indicates activation of platelets and a decreased D-D level is hypothesized to reflect reduced secondary fibrinolytic activity $(27,28)$. All the changes to these molecules observed in the present study were indicated an improvement of thrombosis following paeoniflorin treatment. Although the effect of paeoniflorin was not as potent as aspirin, the practical concentration of paeoniflorin used in the clinic could be adjusted to a level which may be comparable to or stronger than that of aspirin given the low cytotoxicity of paeoniflorin demonstrated by the MTT assay. To achieve this, further comprehensive animal and clinical studies are planned to be conducted in the future. The current study measured the cytotoxic effect of paeoniflorin on HUVECs by an MTT assay. It was demonstrated that the proliferative ability of the cells was increased by paeoniflorin with low cytotoxicity. Although paeoniflorin reduced cell viability in a concentration-dependent manner when the concentration was $>0.5 \mu \mathrm{mol} / 1$, cell viability of HUVECs remained $>70 \%$.

The effect of paeoniflorin on the MAPK signaling pathway has been previously reported. Guo et al (18) demonstrated that chronic treatment with paeoniflorin suppresses the activation of the MAPK pathway, particularly p38 and JNK, and may reduce the production of pro-inflammatory molecules and exert neuroprotective effects during stroke. Although the study by Guo et al (18) and the current study investigated the effects of paeoniflorin on the MAPK pathway, the results demonstrated were quite different. In the present study, the effect of paeoniflorin on MAPK signaling was positive; the phosphorylation of p38 and JNK were increased. The paradox may be caused by the differing concentrations used in the studies. The rats in the previous study received $5 \mathrm{mg} / \mathrm{kg}$ paeoniflorin twice per day for 2 weeks, whereas in the present study, HUVECs received $0.5 \mu \mathrm{mol} / 1$ paeoniflorin for $24 \mathrm{~h}$. This information is important as it may be used to determine the treatment concentration of paeoniflorin if used in the clinic. The dose of paeoniflorin used to treat different diseases should be carefully considered to avoid a negative effect. However, the treatment with JNK and p38 inhibitors exhibited complex effects on the MAPK signaling pathway and expression of uPA. Treatment with SP600125 reduced the phosphorylation of JNK and inhibited the expression of uPA. SB203580, a p38 inhibitor, blocked the phosphorylation of p38 and exhibited an inhibitory effect on the expression level of uPA. These results indicate that the regulation of uPA by paeoniflorin may be dependent on other factors and future comprehensive studies should be conducted to determine the pathways associated with uPA regulation.

In conclusion, paeoniflorin improves the PTS and upregulates uPA via the MAPK signaling pathway. However, this positive effect is dependent on the concentration of paeoniflorin used in the experiment. Different concentrations of paeoniflorin may improve certain diseases and exert a negative effect on others. The results of the present study preliminarily demonstrated that paeoniflorin acts by enhancing the expression of uPA to increase recanlization following thrombosis, which may facilitate the uPA-dependent treatment of thrombosis in the clinic.

\section{References}

1. Italiano JE, Patel-Hett S and Hartwig JH: Mechanics of proplatelet elaboration. J Thromb Haemost 5 (Suppl 1): S18-S23, 2007.

2. Bender M, Eckly A, Hartwig JH, Elvers M, Pleines I, Gupta S, Krohne G, Jeanclos E, Gohla A, Gurniak C, et al: $\mathrm{ADF} / \mathrm{n}$-cofilin-dependent actin turnover determines platelet formation and sizing. Blood 116: 1767-1775, 2010.

3. Ruggeri ZM: Platelets in atherothrombosis. Nature Med 8: 1227-1234, 2002.

4. Stoll G, Kleinschnitz C and Nieswandt B: Molecular mechanisms of thrombus formation in ischemic stroke: Novel insights and targets for treatment. Blood 112: 3555-3562, 2008.

5. Schrör K: Antiplatelet drugs. A comparative review. Drugs 50: 7-28, 1995.

6. Ni H and Freedman J: Platelets in hemostasis and thrombosis: Role of integrins and their ligands. Transfus Apher Sci 28: 257-264, 2003.

7. Barrett NE, Holbrook L, Jones S, Kaiser WJ, Moraes LA, Rana R, Sage T, Stanley RG, Tucker KL, Wright B and Gibbins JM: Future innovations in anti-platelet therapies. Br J Pharmacol 154: 918-939, 2008.

8. Bird JE, Giancarli MR, Allegretto N, Barbera F, Wong P, Schumacher WA, Ogletree ML and Seiffert D: Prediction of the therapeutic index of marketed anti-coagulants and anti-platelet agents by guinea pig models of thrombosis and hemostasis. Thromb Res 123: 146-158, 2008.

9. Northeast AD, Soo KS, Bobrow LG, Gaffney PJ and Burnand KG: The tissue plasminogen activator and urokinase response in vivo during natural resolution of venous thrombus. J Vasc Surg 22: 573-579, 1995.

10. Singh I, Burnand KG, Collins M, Luttun A, Collen D, Boelhouwer B and Smith A: Failure of thrombus to resolve in urokinase-type plasminogen activator gene-knockout mice: Rescue by normal bone marrow-derived cells. Circulation 107: 869-875, 2003.

11. Gossage JA, Humphries J, Modarai B, Burnand KG and Smith A: Adenoviral urokinase-type plasminogen activator (uPA) gene transfer enhances venous thrombus resolution. J Vasc Surg 44: 1085-1090, 2006

12. Aguirre Ghiso JA, Alonso DF, Farías EF, Gomez DE and Bal de Kier Joffè E: Deregulation of the signaling pathways controlling urokinase production. Its relationship with the invasive phenotype. Eur J Biochem 263: 295-304, 1999.

13. Blasi F and Carmeliet P: uPAR: A versatile signalling orchestrator. Nat Rev Mol Cell Biol 3: 932-943, 2002.

14. Lee KH, Hyun MS and Kim JR: Growth factor-dependent activation of the MAPK pathway in human pancreatic cancer: MEK/ERK and p38 MAP kinase interaction in uPA synthesis. Clin Exp Metastasis 20: 499-505, 2003. 
15. Liu SQ, Huang JA, Qin MB, Su YJ, Lai MY, Jiang HX and Tang GD: Sphingosine kinase 1 enhances colon cancer cell proliferation and invasion by upregulating the production of MMP-2/9 and uPA via MAPK pathways. Int J Colorectal Dis 27: 1569-1578, 2012.

16. Benasciutti E, Pagès G, Kenzior O, Folk W, Blasi F and Crippa MP: MAPK and JNK transduction pathways can phosphorylate Sp1 to activate the uPA minimal promoter element and endogenous gene transcription. Blood 104: 256-262, 2004.

17. Nizamutdinova IT, Jin YC, Kim JS, Yean MH, Kang SS, Kim YS, Lee JH, Seo HG, Kim HJ and Chang KC: Paeonol and paeoniflorin, the main active principles of Paeonia albiflora, protect the heart from myocardial ischemia/reperfusion injury in rats. Planta medica 74: 14-18, 2008 .

18. Guo RB, Wang GF, Zhao AP, Gu J, Sun XL and Hu G: Paeoniflorin protects against ischemia-induced brain damages in rats via inhibiting MAPKs/NF- $\kappa \mathrm{B}$-mediated inflammatory responses. PLoS One 7: e49701, 2012

19. National Research Council: Guide for the Care and Use of Laboratory Animals. 8th edition. National Academies Press, Washington, D.C., 1996.

20. Miner $\mathbf{J}$ and Hoffhines A: The discovery of aspirin's antithrombotic effects. Tex Heart Inst J 34: 179-186, 2007.

21. Lijnen HR: Matrix metalloproteinases and cellular fibrinolytic activity. Biochemistry (Mosc) 67: 92-98, 2002.
22. Burnand KG, Gaffney PJ, McGuinness CL, Humphries J, Quarmby JW and Smith A: The role of the monocyte in the generation and dissolution of arterial and venous thrombi. Cardiovasc Surg 6: 119-125, 1998 .

23. Ye J, Duan H, Yang X, Yan W and Zheng X: Anti-thrombosis effect of paeoniflorin: Evaluated in a photochemical reaction thrombosis model in vivo. Planta Med 67: 766-767, 2001.

24. Yoshida A, Nakao S, Kobayashi $M$ and Kobayashi $H$ Flow-mediated vasodilation and plasma fibronectin levels in preeclampsia. Hypertension 36: 400-404, 2000.

25. Pulkkinen MO, Lehto $M$, Jalkanen $M$ and Näntö-Salonen $K$ : Collagen types and fibronectin in the uterine muscle of normal and hypertensive pregnant patients. Am J Obstet Gynecol 149: 711-717, 1984.

26. Lowe GD and Rumley A: Use of fibrinogen and fibrin D-dimer in prediction of arterial thrombotic events. Thromb Haemost 82: 667-672, 1999.

27. Bowen RS, Zhang Y, Gu Y, Lewis DF and Wang Y: Increased phospholipase A2 and thromboxane but not prostacyclin production by placental trophoblast cells from normal and preeclamptic pregnancies cultured under hypoxia condition. Placenta 26: 402-409, 2005.

28. Zhao S, Gu Y, Lewis DF and Wang Y: Predominant basal directional release of thromboxane, but not prostacyclin, by placental trophoblasts from normal and preeclamptic pregnancies. Placenta 29: 81-88, 2008. 